\title{
Maps Which Preserve a Certain Norm Condition between the Exponential Groups of Uniform Algebras
}

\author{
Tatsuya NOGAWA
}

Niigata University

(Communicated by Y. Furuya)

\begin{abstract}
Let $\mathcal{A}_{j}$ be a uniform algebra with a Choquet boundary $C h \mathcal{A}_{j}, j=1,2$. In this paper we prove that if $\phi: \exp \mathcal{A}_{1} \rightarrow \exp \mathcal{A}_{2}$ is a surjection and satisfies the equality

$$
\max \left\{\left\|\frac{\phi(f)}{\phi(g)}-1\right\|_{\infty},\left\|\frac{\phi(g)}{\phi(f)}-1\right\|_{\infty}\right\}=\max \left\{\left\|\frac{f}{g}-1\right\|_{\infty},\left\|\frac{g}{f}-1\right\|_{\infty}\right\}
$$

for any $f, g \in \exp \mathcal{A}_{1}$, then $\phi$ is of the form

$$
\phi(f)(y)= \begin{cases}\phi(1)(y) f(\varphi(y))^{\kappa(y)} & \text { for } y \in K, \\ \phi(1)(y) \overline{f(\varphi(y))^{\kappa(y)}} & \text { for } y \in C h \mathcal{A}_{2} \backslash K\end{cases}
$$

for any $f \in \exp \mathcal{A}_{1}$, where $\kappa$ is a continuous function from $C h \mathcal{A}_{2}$ into $\{1,-1\}, \varphi$ is a homeomorphism from $C h \mathcal{A}_{2}$ onto $C h \mathcal{A}_{1}$ and $K$ is a clopen subset of $C h \mathcal{A}_{2}$.
\end{abstract}

\section{Introduction}

The study of isometries between Banach spaces has a long history dating back to the 1930's. The most fundamental and classical result is the Banach-Stone theorem describing the form of the linear isometries between the Banach spaces of all complex-valued continuous functions on compact Hausdorff spaces. We refer to [1] for an excellent comprehensive treatment of that research area. The celebrated Mazur-Ulam theorem has the same vine, which states that any surjective isometry between normed real-linear spaces is automatically an affine transformation followed by a translation.

Metric-like norm conditions for transformations between subsets of semisimple commutative Banach algebras have recently been studied extensively in the literature. The main reason is that, in many cases, such conditions are strongly encoding that the transformations can be extended to linear and multiplicative maps between the algebras under the consideration. Hence, such properties can be used to characterize algebra isomorphisms. As a reference, we mention the recent survey article [3]. Miura, Honma and Shindo discovered the following

Received November 5, 2014; revised December 10, 2014

Mathematics Subject Classification: 46J10, 47B48

Key words and phrases: Mazur-Ulam theorem, weighted composition operator, uniform algebra 
theorem (a simple case of Theorem 3.1 in [6]); let $\mathcal{A}_{j}$ be a uniform algebra with a Choquet boundary $C h \mathcal{A}_{j}, j=1,2$. If $\phi: \exp \mathcal{A}_{1} \rightarrow \exp \mathcal{A}_{2}$ is a surjection such that the equality

$$
\left\|\frac{\phi(f)}{\phi(g)}-1\right\|_{\infty}=\left\|\frac{f}{g}-1\right\|_{\infty}
$$

holds for any $f, g \in \exp \mathcal{A}_{1}$, then there exist a clopen subset $K$ of $C h \mathcal{A}_{2}$ and a homeomor$\operatorname{phism} \varphi: C h \mathcal{A}_{2} \rightarrow C h \mathcal{A}_{1}$ such that

$$
\phi(f)(y)= \begin{cases}\phi(1)(y) f(\varphi(y)) & \text { for } y \in K, \\ \phi(1)(y) \overline{f(\varphi(y))} & \text { for } y \in C h \mathcal{A}_{2} \backslash K\end{cases}
$$

for any $f \in \exp \mathcal{A}_{1}$. If the equality (1) holds for any $f, g \in \exp \mathcal{A}_{1}$, then we get the equality

$$
\max \left\{\left\|\frac{\phi(f)}{\phi(g)}-1\right\|_{\infty},\left\|\frac{\phi(g)}{\phi(f)}-1\right\|_{\infty}\right\}=\max \left\{\left\|\frac{f}{g}-1\right\|_{\infty},\left\|\frac{g}{f}-1\right\|_{\infty}\right\}
$$

for any $f, g \in \exp \mathcal{A}_{1}$. Surjective maps satisfying the above equation were studied by Hatori, Hirasawa, Miura and Molnár; they proved that such unital maps are group isomorphisms (Theorem 4.3 in [2]). The purpose of this paper is to refine the result by describing the form of such maps.

THEOREM 1. Let $\mathcal{A}_{j}$ be a uniform algebra with a Choquet boundary $C h \mathcal{A}_{j}, j=1,2$. If $\phi: \exp \mathcal{A}_{1} \rightarrow \exp \mathcal{A}_{2}$ is a surjection and satisfies the condition

$$
\max \left\{\left\|\frac{\phi(f)}{\phi(g)}-1\right\|_{\infty},\left\|\frac{\phi(g)}{\phi(f)}-1\right\|_{\infty}\right\}=\max \left\{\left\|\frac{f}{g}-1\right\|_{\infty},\left\|\frac{g}{f}-1\right\|_{\infty}\right\}
$$

for any $f, g \in \exp \mathcal{A}_{1}$, then there exist a continuous function $\kappa: C h \mathcal{A}_{2} \rightarrow\{1,-1\}, a$ homeomorphism $\varphi: C h \mathcal{A}_{2} \rightarrow C h \mathcal{A}_{1}$ and a clopen subset $K$ of $C h \mathcal{A}_{2}$ such that

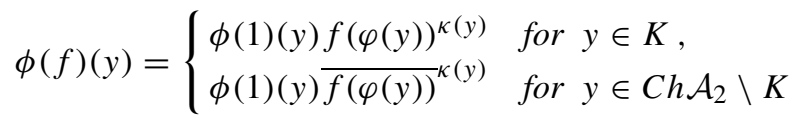

for any $f \in \exp \mathcal{A}_{1}$.

Considering $\kappa \equiv-1$, we note that the form (4) of $\phi$ holds the condition (3) and is not represented by the form (2).

\section{Preliminary}

In this paper $\mathbf{R}, \mathbf{R}_{+}, \mathbf{C}, \mathbf{T}$ are the set of real numbers, the set of non-negative real numbers, the set of complex numbers and the 1-torus of complex numbers, respectively. Recall that a uniform algebra $\mathcal{A}$ on a compact Hausdorff space $X$ is a closed unital subalgebra of complex-valued continuous functions on $X$ which separates the points of $X$. We denote the exponential group of $\mathcal{A}$ by $\exp \mathcal{A}=\left\{e^{u} \in \mathcal{A}: u \in \mathcal{A}\right\}$. The Choquet boundary of $\mathcal{A}$ is denoted by $C h \mathcal{A}$. 
Throughout the paper, let $\mathcal{A}_{j}$ be a uniform algebra and $d_{j}: \exp \mathcal{A}_{j} \times \exp \mathcal{A}_{j} \rightarrow \mathbf{R}_{+}$a map defined by

$$
d_{j}(f, g)=\max \left\{\left\|\frac{f}{g}-1\right\|_{\infty},\left\|\frac{g}{f}-1\right\|_{\infty}\right\}
$$

for every pair $f, g \in \exp \mathcal{A}_{j}, j=1,2$. Note that $d_{j}$ is a metric-like quantity, but not a metric on $\exp \mathcal{A}_{j}$ exactly since the triangle inequality is not satisfied for $d_{j}$ as a simple example shows; $d_{j}(1,3)=2>3 / 2=1+1 / 2=d_{j}(1,2)+d_{j}(2,3)$. We say that a map $\phi: \exp \mathcal{A}_{1} \rightarrow$ $\exp \mathcal{A}_{2}$ is a $d$-preserving map with respect to $d_{1}$ and $d_{2}$ if $d_{2}(\phi(f), \phi(g))=d_{1}(f, g)$ holds for every pair $f, g \in \exp \mathcal{A}_{1}$.

\section{Proof of Theorem 1}

We begin with the following proposition.

PROPOSITION 2. Let $\phi: \exp \mathcal{A}_{1} \rightarrow \exp \mathcal{A}_{2}$ be a surjective d-preserving map with respect to $d_{1}$ and $d_{2}$. Then $\phi$ is a homeomorphism for the topology induced by the uniform norm.

PROOF. It is clear that $\phi$ is an injection, hence it is a bijection. We verify that $\phi$ is a norm continuous map. For any $f \in \exp \mathcal{A}_{1}$ and sequence $\left\{f_{n}\right\}_{n=1}^{\infty}$ of $\exp \mathcal{A}_{1}$ such that $f_{n} \rightarrow f$ as $n \rightarrow \infty$,

$$
\begin{aligned}
\left\|\phi\left(f_{n}\right)-\phi(f)\right\|_{\infty} & =\left\|\left(\phi\left(f_{n}\right) \phi(f)^{-1}-1\right) \phi(f)\right\|_{\infty} \\
& \leq\left\|\phi\left(f_{n}\right) \phi(f)^{-1}-1\right\|_{\infty}\|\phi(f)\|_{\infty} \\
& \leq d_{2}\left(\phi\left(f_{n}\right), \phi(f)\right)\|\phi(f)\|_{\infty} \\
& =d_{1}\left(f_{n}, f\right)\|\phi(f)\|_{\infty} \\
& \rightarrow 0
\end{aligned}
$$

as $n \rightarrow \infty$. Therefore $\phi$ is a norm continuous map. Since $\phi^{-1}$ is a $d$-preserving map with respect to $d_{2}$ and $d_{1}$, in a similar way we infer that $\phi^{-1}$ is norm-continuous.

LeMmA 3. If $\kappa \in \mathbf{T}$ and $u, u^{\prime} \in \mathbf{C}$ with $u \neq u^{\prime}, e^{u}=e^{u^{\prime}}$ and $e^{\kappa u}=e^{\kappa u^{\prime}}$, then $\kappa=1,-1$.

Proof. As $u \neq u^{\prime}$ and $e^{u}=e^{u^{\prime}}$, there is a non-zero integer $n$ with $u-u^{\prime}=2 n \pi i$. In a similar way, there is an integer $m$ with $\kappa\left(u-u^{\prime}\right)=2 m \pi i$. Thus $\kappa=1,-1$ since $\kappa \in \mathbf{T}$.

Theorem 1 is proved by applying Proposition 2 and Lemma 3.

Proof of Theorem 1. Put $\phi_{0}(f)=\phi(1)^{-1} \phi(f)$ for any $f \in \exp \mathcal{A}_{1}$. It is clear that $\phi_{0}(1)=1$ and $\phi_{0}$ is a surjective $d$-preserving map with respect to $d_{1}$ and $d_{2}$. By Theorem 4.3 in [2] and Proposition 2, we have that $\phi_{0}$ is a group isomorphic homeomorphism. For every 
$u \in \mathcal{A}_{1}$, we consider the map

$$
\alpha: \mathbf{R} \ni t \longmapsto \phi_{0}\left(e^{t u}\right) \in \exp \mathcal{A}_{2} .
$$

Since $t \mapsto e^{t u}$ is a norm continuous one parameter group in $\mathcal{A}_{1}$ and $\phi_{0}$ is a group isomorphic homeomorphism, the above map $\alpha$ is a norm continuous one parameter group in $\mathcal{A}_{2}$. Using Proposition 6.4.6 in [7], we obtain the generator $v \in \mathcal{A}_{2}$ of $\alpha$, that is

$$
e^{t v}=\phi_{0}\left(e^{t u}\right), \quad t \in \mathbf{R} .
$$

Defining $T(u)=v$, we obtain a map $T: \mathcal{A}_{1} \rightarrow \mathcal{A}_{2}$ which

$$
\phi_{0}\left(e^{t u}\right)=e^{t T(u)}, \quad t \in \mathbf{R}, u \in \mathcal{A}_{1} .
$$

It is easy to check that $T(0)=0$. We claim that $T$ is a surjection. Considering $\phi_{0}^{-1}$ in the place of $\phi_{0}$, we infer that there exists a map $S: \mathcal{A}_{2} \rightarrow \mathcal{A}_{1}$ such that $\phi_{0}^{-1}\left(e^{t v}\right)=e^{t S(v)}$ holds for every $t \in \mathbf{R}$ and $v \in \mathcal{A}_{2}$. This easily implies that $v=T(S(v))$ holds for every $v \in \mathcal{A}_{2}$. Hence $T$ is a surjection.

We claim that $T$ is an isometry. For every pair $u_{1}, u_{2} \in \mathcal{A}_{1}$, we compute

$$
\begin{aligned}
\frac{1}{|t|} d_{1}\left(e^{t u_{1}}, e^{t u_{2}}\right) & =\frac{1}{|t|} \max \left\{\left\|e^{t\left(u_{1}-u_{2}\right)}-1\right\|_{\infty},\left\|e^{t\left(u_{2}-u_{1}\right)}-1\right\|_{\infty}\right\} \\
& =\max \left\{\left\|\frac{e^{t\left(u_{1}-u_{2}\right)}-1}{t}\right\|_{\infty},\left\|\frac{e^{t\left(u_{2}-u_{1}\right)}-1}{t}\right\|_{\infty}\right\} \\
& \rightarrow\left\|u_{1}-u_{2}\right\|_{\infty}
\end{aligned}
$$

as $t \rightarrow 0$. Similarly, we obtain

$$
\begin{aligned}
\frac{1}{|t|} d_{1}\left(e^{t u_{1}}, e^{t u_{2}}\right) & =\frac{1}{|t|} d_{2}\left(\phi_{0}\left(e^{t u_{1}}\right), \phi_{0}\left(e^{t u_{2}}\right)\right) \\
& =\frac{1}{|t|} d_{2}\left(e^{t T\left(u_{1}\right)}, e^{t T\left(u_{2}\right)}\right) \\
& \rightarrow\left\|T\left(u_{1}\right)-T\left(u_{2}\right)\right\|_{\infty}
\end{aligned}
$$

as $t \rightarrow 0$. It follows that for any pair $u_{1}, u_{2} \in \mathcal{A}_{1}$ we have $\left\|u_{1}-u_{2}\right\|_{\infty}=\left\|T\left(u_{1}\right)-T\left(u_{2}\right)\right\|_{\infty}$. Thus $T$ is an isometry. As $T(0)=0$, the celebrated Mazur-Ulam theorem [4] (c.f [8]) asserts that $T$ is a surjective real-linear isometry between $\mathcal{A}_{1}$ and $\mathcal{A}_{2}$. Applying Theorem 1.1 in [5], there exist a continuous function $\kappa: C h \mathcal{A}_{2} \rightarrow \mathbf{T}$, a homeomorphism $\varphi: C h \mathcal{A}_{2} \rightarrow C h \mathcal{A}_{1}$ and a clopen subset $K$ of $C h \mathcal{A}_{2}$ such that

$$
T(u)(y)= \begin{cases}\kappa(y) u(\varphi(y)) & \text { for } y \in K, \\ \kappa(y) \overline{u(\varphi(y))} & \text { for } y \in C h \mathcal{A}_{2} \backslash K\end{cases}
$$

for any $u \in \mathcal{A}_{1}$. We verify that the range of $\kappa$ is contained in $\{1,-1\}$. Take $y \in C h \mathcal{A}_{2}$ and $u \in \mathcal{A}_{1}$ arbitrarily. We put $u^{\prime}=u+2 \pi i \in \mathcal{A}_{1}$. We consider the case of $y \in K$. Then it is 
clear that $u(\varphi(y)) \neq u^{\prime}(\varphi(y))$ and $e^{u(\varphi(y))}=e^{u^{\prime}(\varphi(y))}$. We have that

$$
e^{T(u)}=\phi_{0}\left(e^{u}\right)=\phi_{0}\left(e^{u^{\prime}}\right)=e^{T\left(u^{\prime}\right)} .
$$

Therefore, we get the equality

$$
e^{\kappa(y) u(\varphi(y))}=e^{\kappa(y) u^{\prime}(\varphi(y))} .
$$

Applying Lemma 3, we obtain $\kappa(y)=1,-1$. Similarly, we infer that $\kappa(y)=1,-1$ in the case of $y \in C h \mathcal{A}_{2} \backslash K$. Thus the range of $\kappa$ is contained in $\{1,-1\}$. Consequently, we deduce that $\phi_{0}$ is of the form

$$
\begin{aligned}
& \phi_{0}(f)(y)=\phi_{0}\left(e^{u}\right)(y) \\
& =e^{T(u)}(y) \\
& = \begin{cases}e^{\kappa(y) u(\varphi(y))} & \text { for } y \in K, \\
e^{\kappa(y) \overline{u(\varphi(y))}} & \text { for } y \in C h \mathcal{A}_{2} \backslash K\end{cases} \\
& = \begin{cases}\frac{f(\varphi(y))^{\kappa(y)}}{f^{\kappa(y)}} & \text { for } y \in K, \\
f(\varphi(y))^{(y)} & \text { for } y \in C h \mathcal{A}_{2} \backslash K\end{cases}
\end{aligned}
$$

for every $f=e^{u} \in \exp \mathcal{A}_{1}$. It is clear that $\phi$ is of the form (4) by the definition of $\phi_{0}$. The proof is complete.

ACKNowledgements. The author would like to express hearty thanks to Professor Osamu Hatori for directing the author to correct an error.

\section{References}

[1] R. J. Fleming and J. E. JAmison, Isometries on Banach spaces: function spaces, Chapman Hall/CRC Monogr. Surv. Pure Appl. Math., 129 Chapman \& Hall/CRC, Boca Raton, 2003.

[2] O. Hatori, G. Hirasawa, T. Miura and L. Molnár, Isometries and maps compatible with inverted Jordan triple products on groups, Tokyo J. Math. 35 (2012), 385-410.

[ 3 ] O. Hatori, S. Lambert, A. Luttman, T. Miura, T. Tonev and R. Yates, Spectral preservers in commutative Banach algebras, Contemp. Math. 547 (2011), 103-123.

[ 4 ] S. MaZur and S. Ulam, Sur les transformations isométriques d'espaces vectoriels normés, C. R. Acad. Sci. Paris 194 (1932), 946-948.

[ 5 ] T. Miura, Real-linear isometries between function algebras, Cent. Eur. J. Math. 9 (2011), 778-788.

[6] T. MiURA, D. Honma and R. Shindo, Divisibly norm-preserving maps between commutative Banach algebras, Rocky Mountain J. Math. 41 (2011), 1675-1699.

[ 7 ] T. W. PALMER, Banach Algebras and the general theory of *-algebras. Vol. I. Algebras and Banach algebras, Encyclopedia of Math. Appl. 49, Cambridge Univ. Press, Cambridge, 1994.

[ 8 ] J. VÄISÄLÄ, A proof of the Mazur-Ulam theorem, Amer. Math. Monthly 110 (2003), 633-635. 
Present Address:

Department of Mathematical Science, Graduate School of Science and Technology, NiIgATA UNIVERSITY,

NiIGATA 950-2181, JAPAN.

e-mail: f12j012h@mail.cc.niigata-u.ac.jp 